\title{
Amalgamative Application of Derivative Operator and Differential Sub- Ordination in Developing Theorems
}

\author{
D. Madhusudana Reddy1*, E. Keshava Reddy2 \\ ${ }^{1}$ Research Scholar, Department of Mathematics,JNTUA,Ananthapuramu, AP, India \\ madhuskd@gmail.com \\ ${ }^{2}$ Professor, Department of Mathematics JNTUA, Anathapuramu, AP, India \\ keshava.maths@jntua.ac.in
}

Article History:Received:11 november 2020; Accepted: 27 December 2020; Published online: 05 April 2021

ABSTRACT:The authors have recently introduced a new generalized derivatives operator $\mu_{\lambda_{1} \lambda_{2}{ }^{n}, m \text {, which }}$ generalized many well-known operators studied earlier by many different authors. The trend of finding new operators such as the differential or the integral operators has attached many researchers and in fact, has become necessary to some of the researchers. The aim of this paper is to the equality relation.

$(1+n) \mu_{\lambda_{1} \lambda_{2}}^{n+1, m} f(z)=z\left(\mu_{\lambda_{1} \lambda_{2}}^{n, m} f(z)\right)^{\prime}+n\left(\mu_{\lambda_{1} \lambda_{2}}^{n+1, m} f(z)\right)$, to discuss some interesting results using the technique of differential subordination. The results include the subordination and the indlusion. Whenever $n=0, \lambda_{2}=0$ we have results given by Oros [10].

Keywords: Analytic function; Hadamard product ( or convolution); Univalent function; Convex function; Derivative operator; Differential subordination; Dominant; Best dominant.

\section{INTRODUCTION AND DEFINITIONS}

Assume that $\mathrm{A}$ is denoting the class of functions of the form $f(z)=z+\sum_{k=2}^{\infty} a_{k} z^{k}, a_{k} \quad$ (1.1) is complex number which are analytic in the open unit disc $U=\{z: z \in \mathbb{C}:|z|<1\}$ on the complex plane $\mathbb{C}$. Assume that $S, S^{*}(\alpha), C(\alpha)(0 \leq \alpha \leq 1)$ indicates the subclasses of A consisting of functions that are univalent, starlike of order $\alpha$ and convex of order $\alpha$ in $U$, respectively. In particular, the classes $S^{*}(0)=S^{*}$ and $C(0)=C$ are the familiar classes of starlike and convex functions in $U$, respectively.

And a function $f \in C(\alpha)$ if $\operatorname{Re}\left[1+\frac{z f^{\prime \prime}}{f^{\prime}}>\alpha\right.$. Furthermore a function $f$ analytic in $U$

is said to be convex if it is univalent and $f(U)$ is convex. Assume that $H(U)$ is the class of holomorphic function in unit disc $U==\{z: z \in \mathbb{C}:|z|<1\}$.

Recognize $\quad A_{n}=\left\{f \in H(U): f(z)=z+a_{n+1}^{z^{n+1}}+\cdots,(z \in U)\right\}$, with $A_{1}=A$. For $\quad a \in \mathbb{C}$ and $n \in \mathbb{N}=$ $\{1,2,3, \ldots\}$

assume that $H[a, n]=\left\{f \in H(U): f(z)=z+a_{n} z^{n}+a_{n+1} z^{n+1}+, \ldots,(z \in U)\right\}$.

Let be given two functions $f(z)=z+\sum_{k=2}^{\infty} a_{k} z^{k}, a_{k}$ and $g(z)=z+\sum_{k=2}^{\infty} b_{k} z^{k}$, analytic in the open unit disc $U=\{z: z \in \mathbb{C}:|z|<1\}$. Then the Hadamard product (or convolution) $f * g$ of two functions $f, g$ is defined by $f(z) * g(z)=(f * g)(z)=z+a_{k} b_{k} z^{k}$. Next, we state basic ideas on subordination. If $f$ and $g$ are analytic in $U$, then the function $f$ is said to be subordinate to $g$, and can be written as $f \prec g$ or $f(z) \prec$ $g(z)(z \in U)$, if and only if there exists the Schwarz function $w$, analytic in $U, w(0)=0$ and $|w(z)|<1$ such that

$f(z)=g(w(z))(z \in U)$, Furthermore if $g$ is univalent in $U$, then $f \prec g$ if and only if $f(0)=g(0)$ and $f(U) \subset$ $g(U)$.

Suppose $\psi: \mathbb{C}^{3} \times U \rightarrow \mathbb{C}$ and let $h$ be univalent in $U$, if $P$ is analytic in $U$ satisfies the (second order) differential subordination $\psi\left[p(z), z p^{\prime}(z), z^{2} p^{\prime \prime}(z) ; z\right) \prec h(z), \quad(z \in U)$,

Then $p$ is known as solution of the differential subordination.

The univalent function $\mathrm{q}$ is known as dominant of the solutions of the differential subordination, or more simply a dominant, if $p \prec q$ for all $p$ satisfying (1.2)dominant $\tilde{q}$ that satisfies

$\tilde{q} \prec q$ for all dominants $q$ of (1.2) is said to be the best dominant of (1.2) . ( Note that the best dominant is unique up to a rotation of $U$ ).

Now, $(x)_{k}$ denotes the Pochhammer symbol (or the shifted factorial)defined by

$(x)_{k}=\left\{\begin{array}{c}\{1 \text { for } k=0, x \in \mathbb{C}\{0\}, \\ x(x+1)(x+2) \ldots(x+k-1) \text { for } k \in \mathbb{N}=\{1,2,3, \ldots,\} \text { and } x \in \mathbb{C} .\end{array}\right.$ studied the generalization derivative operator $\mu_{\lambda_{1} \lambda_{2}}{ }^{n, m}$ which given as the following:

*Corresponding author: D. Madhusudana Reddy

Research Scholar, Department of Mathematics,JNTUA,Ananthapuramu, AP, India madhuskd@gmail.com 
Definition 1. Suppose for $f \in A$ the generalized derivative operator $\mu_{\lambda_{1} \lambda_{2}}{ }^{n, m}$ is defined by $\mu_{\lambda_{1} \lambda_{2}}{ }^{n, m}: A \rightarrow A$ $\mu_{\lambda_{1} \lambda_{2}}{ }^{n, m} f(z)=\varphi_{\lambda_{1} \lambda_{2}}{ }^{m}(z) * R^{n} f(z),(z \in U)$,

$R^{n} f(z)$ denotes the Ruscheweyh derivative operator and $\varphi_{\lambda_{1} \lambda_{2}}^{m}(z)$ analytic function defined by

$$
\begin{gathered}
\varphi_{\lambda_{1} \lambda_{2}}^{m} f(z)=z+\sum_{n=2}^{\infty} \frac{\left(1+\lambda_{1}(k-1)\right)^{m-1} z^{k}}{\left(1+\lambda_{2}(k-1)\right)^{m}}, \quad(z \in U), \\
\mu_{\lambda_{1} \lambda_{2}}{ }_{n}, m f(z)=z+\sum_{n=2}^{\infty} \frac{\left(1+\lambda_{1}(k-1)\right)^{m-1} z^{k} c(n, k) a_{k} z^{k}}{\left(1+\lambda_{2}(k-1)\right)^{m}},(z \in U),
\end{gathered}
$$

where $n, m \in \mathbb{N}_{0}=\{0,1,2, \ldots\}, \lambda_{2} \geq \lambda_{1} \geq 0$ and $c(n, k)=\left[\begin{array}{c}n+k-1 \\ n\end{array}\right]=\frac{(n+1)_{k-1}}{(1)_{k-1}}$. Special cases of this operator include the Ruscheweyh derivative operator in the cases is as follows:

$\mu_{\lambda_{1}, 0}^{n, 1} \equiv \mu_{0,0}^{n, m} \equiv \mu_{0, \lambda_{2}}^{n, m} \equiv R^{n} \quad[14]$, the Salagean derivative operator $\mu_{1,0}^{0, m+1} \equiv S^{n} \quad[15]$, the generalized Ruscheweyh derivative operator $\mu_{\lambda_{1}, 0}^{n, 2} \equiv R_{\lambda}^{n}$ [3], the generalized Salagean derivative operator introduced by A1Oboudi $\mu_{\lambda_{1}, 0}^{0, m+1} \equiv S_{\beta}^{n}$ [2], and the generalized A1-Shaqsi and Darus derivative operator $\mu_{\lambda_{1}, 0}^{0, m+1} \equiv D_{\lambda, \beta}^{n}$ [4]. Now, Let us remind the well known Carlson-Shaffer operator $L(a, c)$ [5] associated with the incomplete beta function $\varphi(a, c ; z)$, defined as follows:

$$
\begin{gathered}
L(z, c)=A \rightarrow A, L(a, c) f(z):=\varphi(a, c ; z) * f(z)(z \in U), \\
\text { where } \varphi(a, c ; z)=z+\sum_{k=2}^{\infty} \frac{(a)_{k-1}}{(c)_{k-1}} z^{k},
\end{gathered}
$$

It is easily seen that $\mu_{\lambda_{1}, 0}^{0,1} f(z)=\mu_{0,0}^{0, m} f(z)=\mu_{0, \lambda_{2}}^{0,0} f(z)=f(z)$ and $\mu_{\lambda_{1}, 0}^{1,1} f(z)=\mu_{0,0}^{1, m} f(z)=\mu_{0, \lambda_{2}}^{0,0} f(z)=$ $z f^{\prime}(z)$ and also $\mu_{\lambda_{1}, 0}^{\alpha-1,0} f(z)=\mu_{0,0}^{\alpha-1, m} f(z)=\mu_{0, \lambda_{2}}^{\alpha-1,0} f(z)$ where $\alpha=1,2,3,, \ldots$.

To prove our results, we need the following equality:

$$
(1+n) \mu_{\lambda_{1}, \lambda_{2}}^{n+1, m} f(z)=z\left(\mu_{\lambda_{1}, \lambda_{2}}^{n, m} f(z)\right)^{\prime}+n\left(\mu_{\lambda_{1}, \lambda_{2}}^{n, m} f(z)\right),(z \in U) .
$$

where $n, m \in \mathbb{N}_{0}=\{0,1,2, \ldots\}, \lambda_{2} \geq \lambda_{1} \geq 0$. Next, we give another definition as follows:

Definition 2. For $n, m \in \mathbb{N}_{0}, \lambda_{2} \geq \lambda_{1} \geq 0$, and $0 \leq \alpha \leq 1$, we let $R_{\lambda_{1}, \lambda_{2}}^{n, m}$ ( $\alpha$ ) denote the class of functions $f \in$ $A$ which satisfy the condition $\operatorname{Re}\left(\mu_{\lambda_{1}, \lambda_{2}}^{n, m} f(z)\right)^{\prime}>\alpha,(z \in U)$.

It is clear that the classes $R_{\lambda_{1}, 0}^{0,1}(\alpha) \equiv R\left(\lambda_{1}, \alpha\right)$ the class of function $f \in A$ satisfying $\operatorname{Re}\left(\lambda_{1} z f^{n}(z)+f^{\prime}(z)>\alpha,(z \in U)\right.$. studied by Ponnusamy [13] and many others.

In the present paper, we shall use the method of differential subordination to derive certain properties of the generalization derivative operator $\mu_{\lambda_{1}, \lambda_{2}}^{n, m} f(z)$. Note that, differential subordination has been studied by various authors, and here we follow similar work done by Oros [9] and Oros and Oros [11].

\section{MAIN RESULTS}

In proving our main results, we need the following Lemmas.

Lemma 1. ([6], p. 71]). Let $h$ be analytic, univalent, convex in $U$, with $h(0)=\alpha, \gamma \neq 0$ and $\operatorname{Re\gamma } \geq 0$. If $p \in$ $H\{a, n]$ and $p(z)+\frac{z p^{\prime}(z)}{\gamma} \prec h(z),(z \in U), p(z) \prec q(z) \prec h(z),(z \in U)$, where $q(z)=\frac{\gamma}{n z^{\frac{\gamma}{n}}} \int_{0}^{z} h(t) t\left(\frac{\gamma}{n}\right)-1 d t$, $(z \in U)$. The function $q$ is convex and is the best $(a, n)-$ dominant.

Lemma 2 ([7]). Let $g$ be a convex function in $U$ and let $h(z)=g(z)+n \alpha z g^{\prime}(z)$, where $\alpha>0$ and $n$ is a positive integer. if $p(z)=g(0)+p_{n} z^{n}+p_{n+1} z^{n+1}+\cdots,(z \in U)$, is holomorphic in $U$ and $p(z)+$ $\alpha z p^{\prime}(z) \prec h(z),(z \in U)$, then $p(z) \prec g(z)$.

Lemma 3 ([8]). Let $f \in A, \operatorname{Re}\left[1+\frac{z f^{n}(z)}{f^{\prime}(z)}\right]>-\frac{1}{2}$, then $2 / z \int_{0}^{z} f(t) d t,(z \in U$ and $z \neq 0)$, belongs to the convex functions. Now we begin with the first result as the following. 
Theorem 1. Let $h(z)=\frac{1+(2 \alpha-1) z}{1+z},(z \in U)$, be convex in $U$, with $h(0)=1$ and $0 \leq \alpha<1$. If $n, m \in \mathbb{N}_{0}, \lambda_{2} \geq$ $\lambda_{1} \geq 0$, and the differential sub-ordination.

$$
\begin{aligned}
& \left(\left(\mu_{\lambda_{1}, \lambda_{2}}^{n+1, m} f(z)\right)^{\prime}<h(z),(z \in U)\right. \\
& \quad\left(\left(\mu_{\lambda_{1}, \lambda_{2}}^{n+1, m} f(z)\right)^{\prime} \prec q(z)=2 \alpha-1+\frac{2(n+1)(1-\alpha) \sigma(n)}{z^{n+1}} \text { where } \sigma\right. \text { is given by } \\
& \sigma(x)=\int_{0}^{z} \frac{t^{x}}{1+t} d t,(z \in u) .
\end{aligned}
$$

The function $q$ is convex and is the best dominant.

Proof. By differentiating (1.3), with respect to $\mathrm{z}$, we obtain

$$
\left(\mu_{\lambda_{1}, \lambda_{2}}^{n+1, m} f(z)\right)^{\prime}=\frac{(1+n)\left(\mu_{\lambda_{1}, \lambda_{2}}^{n, m} f(z)\right)^{\prime}+z\left(\mu_{\lambda_{1}, \lambda_{2}}^{n, m} f(z)^{\prime \prime}\right)}{(1+n)}
$$

Using (2.3) in (2.1), differential subordination (2.1) becomes

$$
\begin{gathered}
\frac{(1+n)\left(\mu_{\lambda_{1}, \lambda_{2}}^{n, m} f(z)\right)^{\prime}+z\left(\mu_{\lambda_{1}, \lambda_{2}}^{n, m} f(z)^{\prime \prime}\right)}{(1+n)}<h(z)=\frac{1+(2 \alpha-1) z}{1+z} \\
p(z)=\left(\mu_{\lambda_{1}, \lambda_{2}}^{n, m} f(z)\right)^{\prime}=\left[z+\sum_{k=2}^{\infty} \frac{\left(1+\lambda_{1}(k-1)^{n-1} c(n, k) a_{k} z^{k}\right.}{\left(1+\lambda_{2}(k-1)\right)^{m}}\right] \\
=1+p_{1} z+p_{2} z^{2}+, \ldots,(p \in H[1,1], z \in U) .
\end{gathered}
$$

Using (2.5) in (2.4), the differential sub-ordination becomes:

$$
\begin{gathered}
p(z)+\frac{z p^{\prime}(z)}{1+n} \prec h(z)=\frac{1+(2 \alpha-1) z}{1+z} \text {. By using Lemma 1, we have } \\
\begin{array}{c}
p(z) \prec q(z)=\frac{(n+1) \int_{0}^{z} h(t) t^{n} d t}{z^{n+1}}=\frac{(n+1) \int_{0}^{z}\left[\frac{1+(2 \alpha-1) t}{1+t}\right] t^{n} d t}{z^{n+1}}\left[\sigma(n)+(2 \alpha-1) \int_{0}^{z} \frac{t^{n+1}}{1+t} d t\right] \\
=(2 \alpha-1)+\frac{2(n+1)(1-\alpha) \sigma(n)}{z^{n+1}} \text { where } \sigma \text { is given by }(2.2), \text { so we get }
\end{array}
\end{gathered}
$$

$\left(\mu_{\lambda_{1}, \lambda_{2}}^{n, m} f(z)\right)^{\prime} \prec q(z)=(2 \alpha-1)+\frac{2(n+1)(1-\alpha) \sigma(n)}{z^{n+1}}$ the functions $q$ is convex and is the best dominant. The proof is complete.

Theorem 2. If $n, m \in \mathbb{N}_{0}, \lambda_{2} \geq \lambda_{1} \geq 0$ and $0 \leq \alpha<1$, then we have $R_{\lambda_{1}, \lambda_{2}}^{n+1, m}(\alpha) \subset R_{\lambda_{1}, \lambda_{2}}^{n+1, m}(\delta)$ where $\delta=$ $(2 \alpha-1)+2(n+1)(1-\alpha) \sigma(n)$. And $\sigma$ is given by $(2.2)$.

Proof. Let $f \in R_{\lambda_{1}, \lambda_{2}}^{n+1, m}(\alpha)$, then from (1.4)we have $\operatorname{Re}\left(\mu_{\lambda_{1}, \lambda_{2}}^{n+1, m} f(z)\right)^{\prime}>\alpha,(z \in U)$. which equivalent is to $\left(\mu_{\lambda_{1}, \lambda_{2}}^{n+1, m} f(z)\right)^{\prime} \prec h(z)=\frac{1+(2 \alpha-1) z}{1+z}$ using Theorem 1, we have $\left(\mu_{\lambda_{1}, \lambda_{2}}^{n, m} f(z)\right)^{\prime}<q(z)=2 \alpha-1+\frac{2(n+1)(1-\alpha) \sigma(n)}{z^{n+1}}$ since $q$ is convex and $q(U)$ is Symmetric with respect to the real axis, we deduce $\operatorname{Re}\left(\mu_{\lambda_{1}, \lambda_{2}}^{n, m} f(z)\right)^{\prime} \prec \operatorname{Req}(1)=\delta=\delta\left(\alpha, \lambda_{1}\right)=2 \alpha-1+2(n+1)(1-\alpha) \sigma(n)$.

For which we deduce $R_{\lambda_{1}, \lambda_{2}}^{n+1, m}(\alpha) \subset R_{\lambda_{1}, \lambda_{2}}^{n, m}(\delta)$. This completes the proof of the Theorem 2 .

Theorem 3. Let $q$ be a convex function in $U$, with $q(0)=1$ and let $h(z)=q(z)+\lambda_{1} z q^{\prime}(z),(z \in$ $U) \quad$ (2.6) then $\left(\mu_{\lambda_{1}, \lambda_{2}}^{n+1, m} f(z)\right)^{\prime} \prec h(z),(z \in U)$ then $\left(\mu_{\lambda_{1}, \lambda_{2}}^{n, m} f(z)\right)^{\prime} \prec q(z)$. and this result is sharp. 
Proof. Let $p(z)=\left(\mu_{\lambda_{1}, \lambda_{2}}^{n, m} f(z)\right)^{\prime}$ using (2.3), differential sub-ordination

(2.6)becomes $p(z)+\frac{z p^{\prime}(z)}{1+n} \prec h(z)=q(z)+\lambda_{1} z q^{\prime}(z),(z \in U)$. Hence $\left(\mu_{\lambda_{1}, \lambda_{2}}^{n, m} f(z)\right)^{\prime} \prec q(z)$,

$(z \in U)$ And the result is sharp. This completes the proof of the theorem. We give a application for

simple

\section{Theorem 3.}

Example 1. For $n=1, m=0, \lambda_{2} \geq \lambda_{1} \geq 0, q(z)=\frac{1+z}{1-z}, f \in A$ and $z \in U$ and applying Theorem 3 , we have

$$
\begin{gathered}
h(z)=\frac{1+z}{1-z}+\lambda_{1} z\left[\frac{1+z}{1-z}\right]^{\prime}=\frac{1+2 \lambda_{1} z-z^{2}}{(1-z)^{2}} \text { After that we find } \\
\left(\mu_{\lambda_{1}, \lambda_{2}}^{1,0} f(z)\right)=z+\sum_{k=2}^{\infty} \frac{c(1, k) a_{k} z^{k}}{1+\lambda_{1}(k-1)},\left(\mu_{\lambda_{1}, \lambda_{2}}^{1,0} f(z)\right)^{\prime} \\
=1+\sum_{k=2}^{\infty} \frac{k^{2} a_{k} z^{k}}{1+\lambda_{1}(k-1)}=f^{\prime}(z) *\left(\varphi_{\lambda_{1}, \lambda_{2}}^{0} f(z)\right)^{\prime} \text { And similarly we shall find } \\
\left(\mu_{\lambda_{1}, \lambda_{2}}^{2,0} f(z)\right)=z+\sum_{k=2}^{\infty} \frac{c(2, k) a_{k} z^{k}}{1+\lambda_{1}(k-1)}=z+\sum_{k=2}^{\infty} \frac{k(k+1) a_{k} z^{k}}{2\left(1+\lambda_{1}(k-1)\right)} \text { Then }
\end{gathered}
$$

$\left(\mu_{\lambda_{1}, \lambda_{2}}^{2,0} f(z)\right)^{\prime}=1+\sum_{k=2}^{\infty} \frac{k^{2}(k+1) a_{k} z^{k-1}}{2\left(1+\lambda_{1}(k-1)\right)}=f^{\prime}(z) *\left(\varphi_{\lambda_{1}, \lambda_{2}}^{0} f(z)\right)^{\prime} *\left[1+\sum_{k=2}^{\infty} \frac{(k+1) z^{k-1}}{2}\right]$ From Theorem 3 we deduce $f^{\prime}(z) *\left(\varphi_{\lambda_{1}, \lambda_{2}}^{0} f(z)\right)^{\prime} *\left[1+\sum_{k=2}^{\infty} \frac{(k+1) z^{k-1}}{2}\right] \prec \frac{1+2 \lambda_{1} z-z^{2}}{(1-z)^{2}}$, implies

$$
f^{\prime}(z) *\left(\varphi_{\lambda_{1}, \lambda_{2}}^{0} f(z)\right)^{\prime} \prec \frac{1+z}{1-z},(z \in U) .
$$

Theorem 4. Let $q$ be a convex function in $U$, with $q(0)=1$ and let $h(z)=q(z)+z q^{\prime}(z)$,

( $z \in U$ ). If $n, m \in \mathbb{N}_{0}, \lambda_{2} \geq \lambda_{1} \geq 0, f \in A$ and satisfies the differential sub-ordination

$$
\left(\mu_{\lambda_{1}, \lambda_{2}}^{n, m} f(z)\right)^{\prime} \prec h(z), \frac{\mu_{\lambda_{1}, \lambda_{2}}^{n, m} f(z)}{z} \prec q(z),(z \in U) \text {. And the result is sharp. }
$$

Proof. $\operatorname{Let} p(z)=\frac{\mu_{\lambda_{1}, \lambda_{2}}^{n, m} f(z)}{z}=\frac{z+\sum_{k=2}^{\infty} \frac{\left(1+\lambda_{1}(k-1)\right)^{m-1} c(n, k)}{\left(1+\lambda_{2}(k-1)\right)^{m}} a_{k} z^{k}}{z}$

$=1+p_{1} z^{1}+p_{2} z^{2}+\cdots(p \in H[1,1]$,

$(z \in U)$.Differentiating, with respect to $z$, we obtain

$\left(\mu_{\lambda_{1}, \lambda_{2}}^{n, m} f(z)\right)^{\prime}=p(z)+z p^{\prime}(z),(z \in U)$. Using

differential sub-ordination (2.10), becomes $p(z)+z p^{\prime}(z) \prec h(z)=q(z)+z q^{\prime}(z)$, using Lemma 2 , we deduce

$p(z) \prec q(z),(z \in U)$, amd using (2.11), we have $\frac{\mu_{\lambda_{1}, \lambda_{2}}^{n, m} f(z)}{z} \prec q(z),(z \in U)$, This proves Theorem 4. We give a simple application for Theorem 4 .

Example 2. For $n=1, m=0, \lambda_{2} \geq \lambda_{1} \geq 0, q(z)=\frac{1}{1-z}, f \in A$ and $z \in U$ and applying Theorem 4, we have

$$
h(z)=\frac{1}{1-z}+z\left(\frac{1}{1-z}\right)^{\prime}=\frac{1}{(1-z)^{2}}
$$

After that we find $\mu_{\lambda_{1}, \lambda_{2}}^{1,0} f(z)=z+\sum_{k=2}^{\infty} \frac{k a_{k} z^{k}}{1+\lambda_{1}(k-1)}, f(z) * \emptyset_{\lambda_{1}, \lambda_{2}}^{0}(z) *\left(z+\sum_{k=2}^{\infty} k z^{k}\right)$.

And from example 1, we have $\left(\mu_{\lambda_{1}, \lambda_{2}}^{1,0} f(z)\right)^{\prime}=f^{\prime}(z) *\left(\emptyset_{\lambda_{1}, \lambda_{2}}^{0}(z)\right)^{\prime}$.

$$
\text { Implies } \frac{f(z) * \emptyset_{\lambda_{1}, \lambda_{2}}^{0}(z) *\left(z+\sum_{k=2}^{\infty} k z^{k}\right)}{z} \prec \frac{1}{(1-z)} \text {. }
$$


Theorem 5. Let $h(z)=\frac{1+(2 \alpha-1) z}{1+z}, \quad(z \in U)$.

Be convex in $U$, with $h(0)=1$ and $0 \leq \alpha<1$. if $n, m \in \mathbb{N}_{0}, \lambda_{2} \geq \lambda_{1} \geq 0, f \in A$ and the differential

$$
\left(\mu_{\lambda_{1}, \lambda_{2}}^{n, m} f(z)\right)^{\prime} \prec h(z)
$$

Then $\frac{\mu_{\lambda_{1}, \lambda_{2}}^{n, m} f(z)}{z}<q(z)=2 \alpha-1+\frac{2(1-\alpha) I n(1+z)}{z}$. The function $q$ is convex and is the best dominant.

$=\frac{z+\sum_{k=2}^{\infty} \frac{\left(1+\lambda_{1}(k-1)\right)^{m-1} c(n, k)}{\left(1+\lambda_{2}(k-1)\right)^{m}} a_{k} z^{k}}{z}$,

Proof. Let $p(z)=\frac{\mu_{\lambda_{1}, \lambda_{2}}^{n, m} f(z)}{z}$,

$=1+p_{1} z+p_{2} z^{2}+\cdots(p \in H[1,1], z \in U)$.

Differentiating (2.14), with respect to $z$, we obtain

$$
\left(\mu_{\lambda_{1}, \lambda_{2}}^{n, m} f(z)\right)^{\prime}=p(z)+z p^{\prime}(z), \quad(z \in U)
$$

Using (2.15), differential subordination (2.13), becomes

$$
p(z)=z p^{\prime}(z) \prec h(z)=\frac{1+(2 \alpha-1) z}{1+z}, \quad(z \in U) .
$$

From Lemma 1, we deduce $p(z) \prec q(z)=\frac{1}{z} \int_{0}^{z} h(t) d t=\frac{1}{z} \int_{0}^{z}\left(\frac{1+(2 \alpha-1) t}{1+t}\right) d t$

$$
=\frac{1}{z}\left[\int_{0}^{z} \frac{1}{1+t} d t+(2 \alpha-1) \int_{0}^{z} \frac{t}{1+t} d t\right]=2 \alpha-1+\frac{2(1-\alpha) \operatorname{In}(1+z)}{z} .
$$

Using (2.14), we have $\frac{\mu_{\lambda_{1}, \lambda_{2}}^{n m} f(z)}{z} \prec q(z)=2 \alpha-1+\frac{2(1-\alpha) \ln (1+z}{z}$.

The proof is complete. From Theorem 5, we deduce the following corollary:

Corollary: if $f \in R_{\lambda_{1}, \lambda_{2}}^{n, m}(\alpha)$ then $\operatorname{Re}\left(\frac{\mu_{\lambda_{1}, \lambda_{2}}^{n, m} f(z)}{z}\right)>2 \alpha-1+2(1-\alpha) \operatorname{In} 2, \quad(z \in U)$.

Proof. Since $f \in R_{\lambda_{1}, \lambda_{2}}^{n, m}(\alpha)$, from Definition $2 \operatorname{Re}\left(\mu_{\lambda_{1}, \lambda_{2}}^{n, m} f(z)\right)^{\prime}>\alpha, \quad(z \in U)$, Which is equivalent to $\left(\mu_{\lambda_{1}, \lambda_{2}}^{n, m} f(z)\right)^{\prime} \prec h(z)=\frac{1+(2 \alpha-1) z}{1+z}$. Using Theorem 5 , we have

$\frac{\mu_{\lambda_{1}, \lambda_{2}}^{n, m} f(z)}{z} \prec q(z)=2 \alpha-1+\frac{2(1-\alpha) I n(1+z)}{z}$ Since $q$ is convex and $q(U)$ is symmetric with respect to the real axis, we deduce $\operatorname{Re}\left(\frac{\mu_{\lambda_{1}, \lambda_{2}}^{n, m}(z)}{z}\right)>\operatorname{Req}(1)=2 \alpha-1+2(1-\alpha) \operatorname{In} 2, \quad(z \in U)$.

Theorem 6. Let, $h \in H(U)$, with $h(0)=1, h^{\prime}(0) \neq 0$ which satisfy the inequality

$$
\operatorname{Re}\left(1+\frac{z h^{\prime \prime}(z)}{h^{\prime}(z)}\right)>-\frac{1}{2}, \quad(z \in U) .
$$

if $n, m \in \mathbb{N}_{0}, \lambda_{2} \geq \lambda_{1} \geq 0, f \in A$ and satisfies the differential subordination

$$
\left(\mu_{\lambda_{1}, \lambda_{2}}^{n, m} f(z)\right)^{\prime}<h(z), \quad(z \in U) .
$$

Then $\frac{\mu_{\lambda_{1}, \lambda_{2}}^{n, m} f(z)}{z} \prec q(z)=\frac{1}{z} \int_{0}^{z} h(t) d t$.

Proof. Let $p(z)=\frac{\mu_{\lambda_{1}, \lambda_{2}}^{n, m} f(z)}{z}=$

$\frac{z+\sum_{k=2}^{\infty} \frac{\left(1+\lambda_{1}(k-1)\right)^{m-1} c(n, k)}{\left(1+\lambda_{2}(k-1)\right)^{m}} a_{k} z^{k}}{z}$,

$=1+p_{1} z+p_{2} z^{2}+\cdots,(p \in H[1,1], z \in U)$.

Differentiating (2.17), with respect to $z$ we have 


$$
\left(\mu_{\lambda_{1}, \lambda_{2}}^{n, m} f(z)\right)^{\prime}=p(z)+z p^{\prime}(z), \quad(z \in U)
$$

Using (2.18), the differential subordination (2.16) becomes $(z)=z p^{\prime}(z) \prec h(z), \quad(z \in U)$ From Lemma 1, we deduce $p(z) \prec q(z)=\frac{1}{z} \int_{0}^{z} h(t) d t$. With (2.17), we obtain

$$
\frac{\mu_{\lambda_{1}, \lambda_{2}}^{n, m} f(z)}{Z} \prec q(z)=\frac{1}{Z} \int_{0}^{z} h(t) d t .
$$

From Lemma 3, we have that the function $q$ is convex, and from Lemma 1, $q$ is the best dominant for subordination (2.16). This completes the proof of Theorem 6.

\section{CONCLUSION}

We remark that several subclasses of analytic univalent functions can be derived using the operator $\mu_{\lambda_{1}, \lambda_{2}}^{n, m}$ and studied their properties.

Acknowledgement: This work is fully supported by UKM-GUP-TMK-07-02-107, Malaysia.

\section{References}

Al-Abbadi, M. H. and M. Darus, 2009. Differential subordination for new generalised derivative operator , (submitted).

Al-Oboudi, F.M, 2004. On univalent functions defined by a generalised Salagean Operator, Into, J. Math. Math. Sci. 27, 1429-1436.

Al-Shaqsi, K. and M. Darus, 2009. On univalent functions with respect to k-summetric points defined by a generalization ruscheweyh derivative operators, Journal of Analysis and Application, 7(1), 53-61.

Al-Shaqsi, K. and M. Darus, 2008. Differential Subordination with generalised derivative operator, Int. J. Comp. Math. Sci, 2(2), 75-78.

Carlson, B. C. and D.B. Shaffer, 1984. Starlike and prestarlike hypergeometric function, SIAM J. Math. Anal. $15,737-745$.

Miller, S. S. and P.T. Mocanu, 2000. Differential subordinations: Theory and applications, in: Series on monographs and textbooks in pure and applied mathematics, 225, Marcel Dekker, New York.

Miller, S. S. and P. T. Mocanu, 1985. On some classes of first-order differential subordinations, Michigan Math. J. 32, 185-195.

Mocanu, P. T. and T. Bulboaca, Gr. St. Salagean, 1999. Teoria geometrica a functiilor univalente, Casa Cartii de Stiinta, Cluj-Napoca.

Oros, G.I, 2005. A class of holomorphic functions defined using a differential operator, General Mathematics, 13(4), 13-18

Oros, G. I, 2003. On a class of holomorphic functions defined by the Ruscheweyh derivative, Int. J. Math. Math. Sci. $65,4139-4144$.

Oros, G. and G. I. Oros, 2004. Differential superordination defined by Salagean operator, General Mathematics, 12(4), 3-10.

Padmanabhan, K. S. and R. Manjini, 1986. Certain applications of differential subordination. Publ. Inst. Math. (N.S.), Tome 39(53), 107-118.

Ponnusamy, S, 1992. Differential subordination and starlike functions, Complex Variables Theory Appl, 19(3), $185-195$

Ruscheweyh, St, 1975. New criteria for univalent functions, Proc. Amer. Math. Soci. Vol. 49, pp. 109-115.

Salagean, G. S, 1983. Subclasses of univalent functions, Lecture Notes in Math. (SpringerVerlag), 1013, 362372. 